\title{
Impacts of COVID-19 on Global Healthcare Management and Research
}

\author{
Stephanie Autore, Santanu De
}

Department of Biological Sciences, Halmos College of Arts and Sciences, Nova Southeastern University, 3301 College Avenue, Fort Lauderdale, Florida 33314, U.S.A.

* Corresponding Author email:

sde@nova.edu

\section{Article History}

Received: 29 May 2021

Revised: 29 October 2021

Accepted: 12 November 2021

Published: 19 November 2021

\section{Student(s)}

- Stephanie Autore

Academic Year: 2020-2021/Fall

Course Level: Bachelor

Course Name: B.S. (Biology)

Course year: Final Year

Mentar(s)

- Dr. Santanu De

\section{A BSTRA CT}

The Coronavirus Infectious Disease 2019 (COVID-19) pandemic has affected most nations at all levels of functioning, individual to governmental. Therefore, it is imperative to evaluate the effects the virus has had on one of the most important socioeconomic global sectors, healthcare. This literature review explores key, recent research and management strategies that have been undertaken in healthcare systems around the world in order to make meaningful attempts at identifying the etiology, diagnosis, and treatment of the disease, and combating the spread of this deadly virus. Healthcare facilities have implemented a variety of changes, all geared towards reducing non-emergency patient visits and preventing transmission of the infection; this includes shifting appointments to online modality via telecommunication use, altering remedial options and procedures, and developing more effective personal protective equipment (PPE). Efforts have also been made to define appropriate treatment methods as well as a potential cure for COVID-19; many vaccines and antiviral drugs have been designed or are continuing to undergo clinical testing, and symptom management approaches are being employed along with careful evaluation or recommendation of already available medications to counter the disease. Our comprehensive analysis provides insights into prominent consequences as well as implications of COVID-19 in contemporary global healthcare research and management, and would help this crucial sector be better prepared for pandemics in the future.

Keywords: COVID-19 pandemic, global impacts, healthcare research and management.

\section{Introduction}

On March 11, 2020, the World Health Organization (WHO) declared the Coronavirus Infectious Disease 2019 (COVID-19) to be a pandemic, affecting multiple countries worldwide, and infecting millions of individuals [1]. The novel strain of this family of viruses, Severe Acute Respiratory Syndrome Coronavirus 2 (SARS-CoV-2), originated in late 2019 within Wuhan, China, with a spread that rapidly developed and disproportionately infected those over 40 years of age [2]. Individuals with comorbid conditions, such as cancer and cardiovascular disease, are especially at risk of infection and have an increased chance of 
mortality due to a weakened immune system [3-5]. The origin of this coronavirus is still relatively unknown; however, there has been speculation that it may have originated from the preparation of bats in a seafood market of Wuhan, China, or the manipulation of another coronavirus within a laboratory [2]. Nonetheless, recent studies have isolated SARS-CoV-2 as the main strain causing the disease, containing an angiotensinconverting enzyme-2 (ACE-2) binding domain, which binds the ACE-2 receptor in various vital organs throughout the body [2]. The wide disbursement of the ACE-2 receptor within the human body may cause infection of COVID-19 to provide a wide variety of symptoms in some patients, making the illness even more complex to treat and manage. The predominant means of transmission of the coronavirus is production of respiratory droplets, which may land on nearby surfaces or travel through the air and be inhaled by another individual [2]. The incubation period of this virus is relatively long, spanning anywhere between 2-14 days prior to symptom development; thus, an individual may be unknowingly infected and could pass on the virus to others quite easily [2].

The COVID-19 outbreak has impacted various areas of research and management of healthcare throughout the globe. Federal and state governments of numerous countries have ordered frequent lockdowns, forcing the workforce to move operations online, and instilling new terms, such as "social distancing" to assist in controlling the contagion. In the realm of healthcare, new research methodologies and management practices have been quickly underway to develop effective treatment options and a possible cure for COVID-19. Preventative protocols have been put in place within healthcare facilities to inhibit the spread of the virus and lessen the risk of transmission among people or communities. The occurrence of COVID19 has transformed many fields of medicine and continues to manifest before the eyes of the world. The newness of the disease, and its constantly evolving status due to repeated mutations of the virus, have rendered it as one of the most influential topics of worldwide concern in recent times. The purpose of this literature review is to highlight recently published, notable reports on the effects of COVID-19 on multifarious aspects of healthcare research and management across the world.

\section{Efforts of Global Healthcare Research and Management}

\subsection{Patient Care Management}

COVID-19 has posed serious risks to healthcare workers and patients in the healthcare setting, especially those who are immunocompromised or require urgent care. This has caused healthcare facilities around the world to make significant changes in ways of providing healthcare efficiently and effectively. Among the initial changes made by healthcare systems following the pandemic's onset was the cancellation of elective surgeries and the implementation of telecommunication for physician appointments [6, 7]. Such processes have been applied in healthcare facilities across Norway, Sweden, the United States, Australia, England, and Egypt. These efforts allow for the reduction in volume of patients within the hospital, reducing possible transmission of the virus, while permitting vital resources to be allocated to patients who are severely ill, whether suffering from COVID-19 or other ailments [6]. Remote monitoring of patient conditions is now being widely utilized; in England, for example, a smartphone app (TeleCheck-AF) is being applied on cardiac patients who may experience atrial fibrillation [8]. The application uses smartphone technology to check the patient's pulse and evaluate any changes for signs of atrial fibrillation; this information is consistently synced with physician charts for examination, enabling continuous remote monitoring of the patient's health [8]. Cardiac patients in England now also have the opportunity to complete rehabilitation programs from home; projects such as "Activate Your Heart", which offer home exercise programs for cardiac patients, are now being offered on video-chat software and have even seen an increase in usage by patients since the start of the pandemic [8].

For individuals who must receive in-patient care due to a severe condition, hospitals and other healthcare facilities have been enforcing strict guidelines within the system to hinder distribution of the virus. Such regulations include the mandate of wearing facial coverings within the hospital setting, restricting, or reducing visitors from entering clinics, and testing for COVID-19 prior to surgery and other procedures [3, 
5]. Some immunocompromised patients who are receiving ongoing treatment, such as chemotherapy for cancer, may have their treatment schedules altered to mitigate the need for in-person visits; however, this depends on the stage and rate of growth of the condition [4, 5]. Egypt has also taken steps to establish separate hospitals focused on treating COVID-19 patients only; this would assist in preventing crosscontamination between hospital departments [3, 9]. Patients are placed in the COVID-19 hospital after being tested for the virus through COVID-19 screening procedures; if a patient tests positive for COVID19, he or she must immediately be transferred to the designated COVID-19 hospital for treatment, while a negative result would allow the patient to be placed in a temporary holding ward until retesting and further placement into the appropriate department are carried out [3].

Social-distancing measures have been established within hospital waiting rooms, with seats placed six feet apart from one another on all sides [3]. Singapore has also taken a special initiative into consideration to develop strategies that limit the spread of the virus in the healthcare setting: the Systems Engineering Initiative for Patient Safety (SEIPS), which provides a holistic view of healthcare - visualizing how each healthcare worker interacts with the environment and associated technologies [9]. With recommendations from SEIPS, Singapore has implemented body temperature checks twice daily for healthcare workers, with the temperature not to exceed $37.5^{\circ} \mathrm{C}$ [9]. Additionally, the use of negative pressure patient-care rooms has been established to prevent air contamination between rooms and other facilities [9].

Many healthcare services have been modifying the way certain medical procedures are performed to prevent aerosolization of respiratory particles; procedures that increase the risk of aerosolization include intubation, extubation, and manual ventilation procedures [10]. However, in some nations, such as the United Kingdom, these procedures may not be recognized as providing an increased risk of transmission, likely increasing the chances of spread of the virus to others [10]. In oncology departments within the United States, procedures that screen for cancer are being modified from endoscopies and bronchoscopies to Computed Tomography (CT)-guided biopsies to reduce the production of respiratory droplets [4]. If highrisk aerosolization procedures cannot be avoided, then stringent precautions are taken; for example, in the cardiology departments within Egypt, intubation procedures are completed prior to entrance into the operating room, ensuring that all nonessential personnel exit the room first [3]. With these efforts, the hospital departments are ensuring that COVID-19 is under control within the realm of healthcare as much as possible.

\subsection{Personal Protective Equipment (PPE)}

The high transmissibility of COVID-19 has caused a rapid increase in the need for the manufacture and use of appropriate personal protective equipment (PPE) to protect healthcare workers. COVID-19 spreads primarily through respiratory droplets, which can travel through the air or land on surfaces prior to being consumed or inhaled by another individual [2]. The length of time that the virus may survive on a surface depends on the material, whether porous or solid; however, this range can vary between a few hours to several days $[2,11]$. Research conducted in the United Kingdom has recognized aerosolized droplets as carrying a significant viral load and posing the highest risk to healthcare staff [10]. Thus, studies have been conducted all over the world to develop proper PPE that will protect the health of frontline workers during this unprecedented time. Such PPE includes the use of face-masks, goggles, face-shields, gloves, and gowns, with a study conducted in Switzerland determining that the most effective coverage is offered by a combined use of all of these forms of PPE [10-12]. There are more stringent guidelines as to what forms of PPE offer the most protection: masks must be either N-95 or FFP2 grade material to block the small size of viral particles $(70-90 \mathrm{~nm})$ that standard surgical masks are ineffective at blocking; masks must also be well-fitted, ensuring complete coverage around the nose and mouth [10]. Furthermore, face-shields must extend below the chin and to the ears, while also allowing space for goggles and a mask to be worn underneath [11]. 
Among the primary concerns regarding the use of PPE is that healthcare workers have been experiencing a shortage of protective equipment, as well as a possible lack of adequate training regarding its use; this poses a significant risk to the health and lives of frontline workers $[10,11]$. Some recommendations, such as the manufacture of face-shields and other forms of PPE within the hospital, have been made to increase the availability and distribution of PPE to healthcare workers [11]. A perspective of the SEIPS model developed in Singapore also suggests that the PPE worn be selected based on the viral load and risk of infection of certain procedures, as this would slow the depletion of supplies [9]. A study in the United Kingdom proposes that a supervisor or "buddy-system" be utilized when dressing in PPE to ensure proper placement and use [10]. In developing countries as well, such as Africa, a shortage of PPE has been noticed; increases in manufacture of these materials and other vital medical equipment has been progressing [13]. Due to the rough terrain and difficult-to-reach locations of some medical clinics and hospitals in Africa, there has been an operation of utilizing drones to transport material quickly and efficiently, greatly reducing the transport time of imperative medical supplies [13]. Without the use of PPE, the spread of the coronavirus would increase exponentially and put the lives of populations in potential danger, making the virus even more difficult to control.

\subsection{Treatment Options}

The symptoms of COVID-19 can be discomforting and debilitating, with the most common consisting of dry cough, fever, and shortness of breath, which may manifest into pneumonia and develop further complications that may lead to death $[2,14]$. Furthermore, the symptoms experienced by children can be different than those experienced by adults and tends to take the form of another ailment: Pediatric Multisystem Inflammatory Syndrome, characterized by fever, cough, edema, and decreased heart functioning [15]. Due to the virus's novelty, at the disease's outset there was no vaccine or medication available to cure the illness; however, extensive and rapid several clinical trials have been conducted on individuals who suffer from COVID-19. England has made many attempts to establish a proper treatment option for COVID-19; among these are the use of medications such as Chloroquine and Hydroxychloroquine $[16,17]$. Chloroquine and Hydroxychloroquine have been utilized to treat a variety of conditions, such as malaria, arthritis, SARS, and MERS; they function by conducting a neutralization reaction that will deactivate acids $[16,17]$. If a cell is equipped with chloroquine/hydroxychloroquine and becomes invaded by a virus, it will be able to neutralize the acidic environment required for the virus to survive [16]. Studies in England have also found Remdesivir to be a promising remedial approach against COVID-19, as it functions to inhibit the replication processes of viruses [16, 17].

One of the recent treatment methods explored in Europe has been the use of convalescent plasma, which is obtained from an individual who survived a virus and contains antibodies for fighting against the viral particles [18]. Convalescent plasma is normally utilized during epidemic situations, in which there is a lack of vaccination or effective cure for an illness, as seen during the Spanish influenza of 1918-1920 [18]. Studies administered in England and Wuhan, China found that when administered to an individual with COVID19, convalescent plasma works best in the early stages of viral infection, as the antibodies IgG and IgM are at their highest levels $[18,19]$. Convalescent plasma also does not have any known adverse side-effects and allows for protection of further infection for up to a few months after injection [18, 19]. Besides, clinical trials conducted in China found that the use of convalescent plasma also assists in the functioning of the cardiovascular and immune systems, providing increased oxygenation to tissues, and decreases inflammation and viral load, with a lesser risk of being admitted to the Intensive Care Unit $[18,19]$. While convalescent plasma has been quite effective in treating COVID-19, there has been an issue that is difficult to overcome: the supply of convalescent plasma to is too low to assist in treating every individual who is infected; this is because far more individuals are ill with the coronavirus than those who have fully recovered [18]. It is also imperative to ensure that the convalescent plasma is devoid of any other pathogens and impurities; the process of filtering plasma mirrors the screening process of blood transfusions: all contaminations must be removed via the use of Pathogen Reduction Technology (PRT) [18]. Countries 
such as Australia and New Zealand, however, do not have access to such technologies, rendering the need for stringent donor guidelines [5].

The year 2021 has seen mass rollout of COVID-19 vaccine in several nations to serve a high proportion of the adult population, and currently approved vaccines have been significantly effective in countering disease's severity and mortality rates; however, equitable vaccine access around the globe continues to be challenge [20]. Concerns about vaccination could be eliminated by strengthening awareness of COVID-19 vaccine among health care workers (HCWs), especially female HCWs under 30 years with no history of influenza vaccination [21]. A recent analysis has reviewed the development of COVID-19 vaccines, underlining the efficacy and vaccination reactions of the authorised vaccines, as well as the mechanisms, storage, and dosage specification of vaccine candidates at the advanced stage of development while also considering potential challenges [22]. In a quantitative synthesis of phase III trials to detect modifying factors that might potentially influence the clinical efficacy of COVID-19 vaccines, network meta-analysis provided the following rank of effectiveness: BNT162b2 $\simeq$ mRNA-1273 > Sputnik V >> AZD1222 [23]. In response to the overwhelming pace and volume of global vaccine research, an online, interactive vaccine tracker has been developed, hosted by the Vaccine Centre at the London School of Hygiene and Tropical Medicine, UK [24]. Clinical trials for COVID-19 vaccination had been underway since 2020 in many countries, including a few that conducted animal trials for effectiveness and one study expedited towards human trials [17]. The adenovirus vaccine, among others, has entailed the application of an inactivated form of the coronavirus and the use of plasmid DNA to fight the virus prior to infection [25]. Furthermore, results of this study have suggested a potential roadmap for designing universal vaccine(s) against the broad range of coronaviruses by continuing exploration of the B-cell repertoire to identify cross-reactive epitopes. Despite the development of diverse COVID-19 vaccines worldwide, owing to concerns around their relative safety and efficacy coupled with vaccination hesitancy among people, healthcare workers are advised to continue to utilize symptom management techniques combined with increased availability and affordability of testing services to care for patients, including asymptomatic carriers.

\subsection{Management of COVID-19 Symptoms}

According to the National Institute for Health and Care Excellence (NICE), treatment of the common symptoms of COVID-19 depends on the severity of the condition; for example, a light cough and sore throat may be alleviated with honey, while a more powerful cough may render the need of a codeine medication [14]. The administration of paracetamol has been found to be most effective in reducing fevers of those who are ill with COVID-19 [14]. In order to manage shortness of breath, certain breathing techniques have been effective, such as "pursed-lip breathing" as well as medications like antiemetics [14]. Furthermore, ventilators may be required if the patient has severe difficulty breathing on their own; however, there have been shortages of ventilators in major hospitals due to the large influx of COVID-19 patients [26]. Thus, hospitals in Brazil have been proposing the use of anesthesia machines to substitute the use of ventilators whenever necessary [26].

COVID-19 can also take a psychological toll, increasing levels of anxiety and stress among the patient, family, and healthcare professionals [14, 27]. In a study conducted in China, there were higher incidences of stress and anxiety by healthcare workers due to the COVID-19 pandemic, with nurses experiencing worse symptoms than physicians; this also coincides with the experiences of hospitals in Greece [27]. The stress experienced by the individual may also manifest into physical discomfort viz; difficulty in breathing, hypoxia, and constipation [14]. Among the most common corrective actions for anxiety and stress during the pandemic is communication; patients, families, and healthcare workers must address any concerns regarding prognosis and treatment $[14,27]$. The administration of anti-anxiety medications, such as benzodiazepine may also be helpful in easing anxiety [14]. Adjusting to the new way of life the pandemic has brought along is challenging and may take some time to become accustomed to; however, through 
interpersonal interactions regarding the virus and coping measures, people may be able to remain calm and make appropriate decisions regarding their health safety and management.

\section{Discussion}

COVID-19 has led to remarkable reforms in healthcare research and management at multiple levels worldwide. In the United States and other countries around the world, most non-essential healthcare activities were cancelled or postponed, and governments had to declare a shut-down of in-person related economic activities in attempts to curb the spread of the virus. Several medical facilities have moved their functions online via remote services, such as Zoom meetings on Zoom, Skype, or other digital platforms. Students, faculty, and college administrators had already been facing a multitude of barriers in STEM education, given its changing landscape in recent times [28, 29]. In this context, COVID-19 impacted not only healthcare research and management but education in healthcare and STEM (among most other academic sectors), necessitating evidence-based strategies for effective pedagogy and assessment of STEM courses conducted online because of the pandemic [30-38].

Though absence of in-person interactions have made people face inconveniences often during these unusual circumstances, conscious efforts to inhibit the transmission of the virus must continue to be exercised by everyone to ensure health and wellness of themselves and others. These can be achieved by increased exposure to knowledge about the virus: how it functions or spreads, and how surfaces can be properly disinfected; in other words, the population must increase their health literacy [39]. Health literacy involves gathering accurate information in order to apply it to make judicious, informed decisions regarding one's health [39]. Administrative authorities of many countries have been taking steps to promote health literacy; however, those would not be successful without active cooperation of the general population to educate themselves and their families and children about the importance of proper hygiene, cleanliness, and regular sanitary habits. Given the COVID-19-based global socioeconomic crisis, knowledge of food safety and scientific research undertaken to meet the rising demands of food and nutrition, especially in developing countries, need to be acquired and disseminated [40-43].

Greater efficiency of research and management among healthcare professionals is not possible without improving healthcare/science education in schools and colleges. In order to meet the aggravated COVID19-based demands of e-pedagogy, educators and academic administrators may consider suitable instances of empowering the youth with awareness and interest in science through modern online tools such as costeffective mobile applications with real-world relevance at middle/high school or college levels [44-47], fostering engagement and learning among healthcare science students by developing and implementing virtual classrooms [48], organizing and supporting digital leadership forums [49], employing new means of course assessment such as peer-video-blogs [50], and may need to restructure novel assessment strategies recently designed for research-based science courses [51-55].

On the other hand, COVID-19 has caused heightened health safety concerns and stress among people, so much so, that individuals are worried to even enter a healthcare facility. Due to this, many hematology departments are experiencing lower yields of blood samples donated or available for transfusions $[4,56]$. On account of this shortage, hospitals in Africa have been implementing Patient Blood Management (PBM) techniques to decrease the need for blood transfusions whenever possible, which consist of increasing erythropoiesis and reducing the risk of blood loss [56]. Hematology departments in Australia are addressing this issue through administration of supplements that enhance the functioning of erythrocytes, such as iron, vitamin B12, and folic acid [5]. There is hope that, with such continued endeavors, complemented by fostering health literacy among the masses, the pandemic will be brought under control and the health of global populations will flourish again.

\section{Conclusions}

The impacts that the COVID-19 pandemic has had on healthcare research and management have been intense and alarming. Healthcare professionals have been applying their knowledge of pre-existing diseases 
and viruses to their immediate practice in the field of medicine, including major shifts to telecommunication in multiple relevant domains, and altering treatment protocols to inhibit transmission of the infection through respiratory droplets. Significant efforts have been made to protect essential or frontline healthcare workers, patients, and the surrounding population with the use of PPE, while assessing the risk of contamination with appropriate combinations of protective wear. COVID-19 has not only influenced individuals physically, but also socially, economically, emotionally, and psychologically, necessitating timely and widespread communication of healthcare research/management updates to help people avoid stress and uncertainty during the pandemic, and to provide the population and healthcare workers with accurate information concerning the virus. While the novel coronavirus presents ambiguities regarding proper remedial measures to prevent the infection, control the symptoms, and cure the disease, various clinical research investigations are being performed throughout the world to determine the quickest and most effective form of care, with minimal side-effects. Furthermore, the history of earlier pandemics may provide some optimism that the spread of the coronavirus will eventually be under control and the case-curves would flatten based on successful production and dissemination of vaccines through validated human clinical trials coupled with generation of herd immunity in populations, improved antiviral medications, and cutting-edge treatment procedures. This paper encapsulates recently published scientific literature citing critical developments in healthcare research and management practices in response to COVID-19. Numerous fresh endeavours have been continuing across the world including faster and more accurate diagnostic strategies to detect the infection, design of vaccines, and better antiviral drugs for prevention and potential cure, to name a few. Therefore, a possible limitation of the present literature review could be its inability to provide an exhaustive list of all relevant reports on the impact of COVID-19 on global health research and management. Nonetheless, the study could serve as a foundation for pertinent future projects; for instance, improvements aimed at ensuring cost-effective, personalized healthcare among underprivileged and minority populations and people from socioeconomically challenged backgrounds or difficult geographical locations in developing countries, on a case-by-case basis. Moreover, additional healthcare management and research on COVID-19-associated illnesses and the impact on pediatric patients is necessary for a cohesive understanding of the novel coronavirus and its implications in patients of specific age-groups.

\section{Declarations}

\subsection{Study Limitations}

Owing to the ongoing status of the pandemic, new studies from various countries are continuing to be published on the topic. Hence, this literature review is not exhaustive of all possible COVID-19-related implementations of health research and management practices yet accomplished around the world. Recommended further research could focus on age-appropriate clinical interventions, with possible emphasis on minorities or underprivileged communities in developing countries, while also taking into consideration better treatment strategies and healthcare policy solutions regarding conditions associated with COVID-19; for example, Pediatric Multisystem Inflammatory Syndrome.

\section{$5.2 \quad$ Competing Interests}

The authors declare that there were no conflicts of interest involved in this literature review.

\section{How to Cite this Article:}

S. Autore and S. De, "Impacts of COVID-19 on Global Healthcare Management and Research", Adv. J. Grad. Res., vol. 11, no. 1, pp. 52-60, Nov. 2021. https://doi.org/10.21467/ajgr.11.1.52-60 


\section{References}

[1] D. Cucinotta and M. Vanelli, "WHO Declares COVID-19 a Pandemic," Acta Biomed, vol. 91, no. 1, pp. 157-160, Mar 192020

[2] F. A. Rabi, M. S. Al Zoubi, G. A. Kasasbeh, D. M. Salameh, and A. D. Al-Nasser, "SARS-CoV-2 and Coronavirus Disease 2019. What We Know So Far," Pathogens, vol. 9, no. 3, Mar 202020.

[3] S. Shaheen et al., "Rapid guide to the management of cardiac patients during the COVID-19 pandemic in Egypt: "a position statement of the Egyptian Society of Cardiology"," Egypt Heart J, vol. 72, no. 1, p. 30, May 272020.

[4] P. Cinar et al., "Safety at the Time of the COVID-19 Pandemic: How to Keep our Oncology Patients and Healthcare Workers Safe," J Natl Compr Canc Netw, pp. 1-6, Apr 152020.

[5] R. Weinkove et al., "Managing haematology and oncology patients during the COVID-19 pandemic: interim consensus guidance," Med J Aust, vol. 212, no. 10, pp. 481-489, Jun 2020.

[6] R. J. Lindeman, M. Sund, J. Lofgren, T. Basso, and K. Soreide, "Preventing spread of SARS-CoV-2 and preparing for the COVID19 outbreak in the surgical department: perspectives from two Scandinavian countries," J Surg Case Rep, vol. 2020, no. 5, p. rjaa131, May 2020.

[7] F. C. Hojaij, L. A. Chinelatto, G. H. P. Boog, J. A. Kasmirski, J. V. Z. Lopes, and F. M. Sacramento, "Surgical Practice in the Current COVID-19 Pandemic: A Rapid Systematic Review," Clinics (Sao Paulo), vol. 75, p. e1923, 2020.

[8] L. Klompstra and T. Jaarsma, "Delivering healthcare at distance to cardiac patients during the COVID-19 pandemic: Experiences from clinical practice," Eur J Cardiovasc Nurs, p. 1474515120930558, Jun 22020.

[9] W. Hoe Gan, J. Wah Lim, and D. Koh, "Preventing intra-hospital infection and transmission of COVID-19 in healthcare workers," Saf Health Work, Mar 242020.

[10] J. B. T. Herron, A. G. C. Hay-David, A. D. Gilliam, and P. A. Brennan, "Personal protective equipment and Covid 19- a risk to healthcare staff?," Br J Oral Maxillofac Surg, vol. 58, no. 5, pp. 500-502, Jun 2020.

[11] C. Wesemann et al., "3-D Printed Protective Equipment during COVID-19 Pandemic," Materials (Basel), vol. 13, no. 8, Apr 242020.

[12] A. Haleem, M. Javaid, R. Vaishya, and S. G. Deshmukh, "Areas of academic research with the impact of COVID-19," Am J Emerg Med, vol. 38, no. 7, pp. 1524-1526, Jul 2020.

[13] C. Ihekweazu and E. Agogo, "Africa's response to COVID-19," BMC Med, vol. 18, no. 1, p. 151, May 222020.

[14] H. National Institute for, N. H. S. E. Care Excellence in collaboration with, and N. H. S. Improvement, "Managing COVID-19 symptoms (including at the end of life) in the community: summary of NICE guidelines," BMJ, vol. 369, p. m1461, Apr 202020.

[15] M. P. Deza Leon et al., "COVID-19-Associated Pediatric Multisystem Inflammatory Syndrome," J Pediatric Infect Dis Soc, vol. 9 , no. 3, pp. 407-408, Jul 132020.

[16] E. Mahase, "Covid-19: what treatments are being investigated?," BMJ, vol. 368, p. m1252, Mar 262020.

[17] A. Mendes, "Research towards treating COVID-19," Br J Community Nurs, vol. 25, no. 4, pp. 204-205, Apr 22020.

[18] B. L. Brown and J. McCullough, "Treatment for emerging viruses: Convalescent plasma and COVID-19," Transfus Apher Sci, vol. 59, no. 3, p. 102790, Jun 2020.

[19] M. Ye et al., "Treatment with convalescent plasma for COVID-19 patients in Wuhan, China," J Med Virol, Apr 152020.

[20] J. S. Tregoning, K. E. Flight, S. L. Higham, Z. Wang, and B. F. Pierce, "Progress of the COVID-19 vaccine effort: viruses, vaccines and variants versus efficacy, effectiveness and escape," Nat Rev Immunol, vol. 21, no. 10, pp. 626-636, Oct 2021.

[21] C. Luo et al., "Intention to COVID-19 vaccination and associated factors among health care workers: A systematic review and metaanalysis of cross-sectional studies," Am J Infect Control, vol. 49, no. 10, pp. 1295-1304, Oct 2021.

[22] Y. Yan et al., "The COVID-19 Vaccines: Recent Development, Challenges and Prospects," Vaccines (Basel), vol. 9, no. 4, Apr 5 2021.

[23] L. Calzetta, B. L. Ritondo, A. Coppola, M. G. Matera, N. Di Daniele, and P. Rogliani, "Factors Influencing the Efficacy of COVID19 Vaccines: A Quantitative Synthesis of Phase III Trials," Vaccines (Basel), vol. 9, no. 4, Apr 12021.

[24] M. Shrotri, T. Swinnen, B. Kampmann, and E. P. Parker, "An interactive website tracking COVID-19 vaccine development," The Lancet Global Health, vol. 9, no. 5, pp. e590-e592, 2021.

[25] H. Lv, N. C. Wu, and C. K. P. Mok, "COVID-19 vaccines: Knowing the unknown," Eur J Immunol, vol. 50, no. 7, pp. 939-943, Jul 2020.

[26] M. L. A. Torres, F. A. T. Canhisares, and V. C. Quintao, "Management of $\mathrm{CO} 2$ absorbent while using the anesthesia machine as a mechanical ventilator on patients with COVID-19," Braz J Anesthesiol, Apr 222020.

[27] K. Tsamakis et al., "COVID-19 pandemic and its impact on mental health of healthcare professionals," Exp Ther Med, vol. 19, no. 6, pp. 3451-3453, Jun 2020.

[28] S. De and G. Arguello, "STEM Education in College: An Analysis of Stakeholders' Recent Challenges and Potential Solutions," FDLA Journal, vol. 5, 2020, Art. no. 9.

[29] G. Arguello, S. De, and S. Orta, "An Analysis of STEM Education at the College Level: Stakeholders' Perspectives," Biology Faculty Proceedings, Presentations, Speeches, Lectures, Conference Presentation 2020, Art. no. 426.

[30] S. Autore, J. Hallet, M. Hoang, and S. De, "Impact of COVID-19 on Global Education and Research in Healthcare and STEM," Presentation 2020. Trick to the Treat of Internships and Research, Nova Southeastern University

[31] S. De, "Impacts of the COVID-19 Pandemic on Global Education," Book Chapter pp. 84-94, 2020. Royal Book Publishing

[32] S. Autore, J. Hallett, M. Hoang, and S. De, "Navigating COVID-19-based Challenges to Global Education, Research, and Management in Healthcare and STEM," Biology Faculty Proceedings, Presentations, Speeches, Lectures, Conference Presentation 2021, Art. no. 430.

[33] J. Hallett and S. De, "Effects of COVID-19 on Education in Healthcare and STEM," AIJR Preprints, vol. 275, no. 1, 2020

[34] M. Hoang, J. Hallett, S. Autore, and S. De, "Education, research, and management in STEM and healthcare: global impacts of COVID-19," Biology Faculty Proceedings, Presentations, Speeches, Lectures, Conference Presentation 2021, Art. no. 432. 
[35] J. Hallett, S. Autore, M. Hoang, and S. De, "COVID-19-based Challenges and Countermeasures in Education, Research, and Management in Healthcare and STEM," Biology Faculty Proceedings, Presentations, Speeches, Lectures, Conference Presentation 2021, Art. no. 431.

[36] S. Autore and S. De, "Effects of COVID-19 on Global Healthcare Research and Management," AIJR Preprints, vol. 314, no. 1, 2021.

[37] M. Hoang and S. De, "Effects of COVID-19 on Global Research in STEM," AIJR Preprints, Preprint no. 1, 2021, Art. no. 331.

[38] S. De and G. Arguello, "Key Strategies for Effective Pedagogy and Assessment of College STEM Courses Online during COVID19," Biology Faculty Proceedings, Presentations, Speeches, Lectures, Conference Presentation 2021, Art. no. 445.

[39] L. Paakkari and O. Okan, "COVID-19: health literacy is an underestimated problem," The Lancet Public Health, vol. 5, no. 5, pp. e249-e250, 2020.

[40] S. De, "Food safety: Steps of rising concern," Everyman's Science, vol. XLV, no. 4, pp. 219-222, 2010.

[41] S. De, "Strategies of Plant Biotechnology to Meet the Increasing Demand of Food and Nutrition in India," International Annals of Science, vol. 10, no. 1, pp. 7-15, 2020.

[42] S. De, "Identification and Cloning of Putative Serine Protease Inhibitor (Serpin) Genes in Rice (Oryza sativa) and a Preliminary Approach to Generate RNAi using the Cloned Sequences," Preprint pp. 1-33, 2019, Art. no. 978.

[43] S. De and S. Bandyopadhyay, "Molecular Taxonomy: An Approach Based on Molecular Markers," Science and Culture, vol. 74, pp. 397-496, 2008.

[44] S. De and V. Nethi, "The potential of socio-biologically relevant mobile apps to attract girls to STEM," Biology Faculty Proceedings, Presentations, Speeches, Lectures, Conference Presentation 2019, Art. no. 334.

[45] V. Nethi and S. De, "The Potential of Socio-biologically Relevant Mobile Applications to Attract Girls to STEM," FDLA Journal, vol. 4, no. 1, 2019, Art. no. 4.

[46] V. Nethi and S. De, "Use of Science Mobile Apps among Undergraduate Science Students and Its Impact on Their Interest and Learning," Biology Faculty Proceedings, Presentations, Speeches, Lectures, Conference Presentation 2020, Art. no. 427.

[47] S. De and V. Nethi, "Impact of Science Mobile Applications on Interest and Learning Among Undergraduate Science Students," Quarterly Review of Distance Education, vol. 21, no. 4, pp. 37-50, 2020.

[48] S. De and G. Cavanaugh, "Navigating Healthcare Science Student Learning and Engagement Through Implementation of a Virtual Classroom," Biology Faculty Proceedings, Presentations, Speeches, Lectures, Conference Presentation 2020, Art. no. 419.

[49] S. De, "Anatomy and Physiology Breakout Session/Focus Group, Pearson's Digital Leadership Forum, Orlando, FL, USA," Biology Faculty Proceedings, Presentations, Speeches, Lectures, Panel Discussion 2020, Art. no. 420.

[50] E. Luyegu and S. De, "Peer-Video-Blog Assessment: An Innovative Approach to Assessment," Biology Faculty Proceedings, Presentations, Speeches, Lectures, Conference Presentation 2020, Art. no. 428.

[51] B. Kim, R. Muchintala, O. Haughton, S. De, and A. Sikora, "Novel Assessment Strategies for Biochemistry Courses Using the Research-Based Biochemistry Authentic Student Inquiry Lab (BASIL) Model," Biology Faculty Proceedings, Presentations, Speeches, Lectures, Conference Presentation 2020, Art. no. 423.

[52] B. Kim, O. Haughton, R. Muchintala, S. De, and A. Sikora, "Design of research-based assessment strategies for a biochemistry CURE using published learning outcomes," Biology Faculty Proceedings, Presentations, Speeches, Lectures, Conference Presentation 2020, Art. no. 425.

[53] A. Kapil, N. Pathak, A. Sikora, and S. De, "Assessment of Student Mastery of Anticipated Learning Outcomes During a BlendFlex STEM CURE Using a Combination of Self-reported and Empirical Analysis," Biology Faculty Proceedings, Presentations, Speeches, Lectures, Conference Presentation 2021, Art. no. 429.

[54] A. Kapil, S. De, and A. Sikora, "Analysis of Student Learning Gains in a Biochemistry CURE course during the mandatory COVID19 shift to online learning," Biology Faculty Proceedings, Presentations, Speeches, Lectures, Conference Presentation 2021, Art. no. 434.

[55] N. Pathak, M. Tariq, S. De, and A. Sikora, "Analysis of student mastery of anticipated learning outcomes during a BlendFlex STEM CURE using a combination of self-reported and empirical analysis," Biology Faculty Proceedings, Presentations, Speeches, Lectures, Conference Presentation 2021, Art. no. 433.

[56] C. L. Barrett, "Primary healthcare practitioners and patient blood management in Africa in the time of coronavirus disease 2019: Safeguarding the blood supply," Afr J Prim Health Care Fam Med, vol. 12, no. 1, pp. e1-e3, May 212020.

Publish your books with AIJR publisher-

$\checkmark \quad$ Publish with ISBN and DOI.

$\checkmark \quad$ Publish Thesis/Dissertation as Monograph.

$\checkmark$ Publish Book Monograph.

$\checkmark$ Publish Edited Volume/ Book.

$\checkmark \quad$ Publish Conference Proceedings

$\checkmark \quad$ Retain full copyright of your books.

Submit your manuscript at books.aijr.org
Publish your research article in AIJR journals-

$\checkmark \quad$ Online Submission and Tracking

$\checkmark$ Peer-Reviewed

$\checkmark$ Rapid decision

$\checkmark \quad$ Immediate Publication after acceptance

$\checkmark$ Articles freely available online

$\checkmark \quad$ Retain full copyright of your article.

Submit your article at journals.aijr.org 